\title{
基于深度图像的手部姿态估计综述
}

\author{
车云龙 ${ }^{1)}$, 齐越 $\left.1,2,3\right)^{*}$ \\ ${ }^{1)}$ (北京航空航天大学虚拟现实国家重点实验室 北京 100191) \\ 2) (鹏城实验室 深圳 518000) \\ 3) (北京航空航天大学青岛研究院 青岛 266100) \\ (qy@buaa.edu.cn)
}

\begin{abstract}
摘 要: 基于深度图像的手部姿态估计是人机交互和虚拟现实领域的一个重要研究问题. 对近些年来该领域的研究 工作进行总结和梳理. 首先, 简述了该问题的定义以及所面临的主要难点, 并总结了常用的深度相机、数据集和评价 指标; 其次，将该领域内的工作分为 3 个类别并依次进行回顾，其中包括基于模型驱动的方法、从数据集中学习映射 函数的基于数据驱动的方法以及同时结合了前两者的混合方法，在叙述过程中，着重介绍了其解决的科学问题以及 仍存在的缺陷; 最后, 从算法的准确性、适用性和鲁棒性 3 个角度对这些工作分别进行进一步的分析, 并对未来的研 究方向进行展望.
\end{abstract}

关键词：深度图像；手部姿态估计；手部追踪；深度学习；人机交互

中图法分类号: TP391.41 DOI: 10.3724/SP.J.1089.2021.18788

\section{A Survey on Depth Based Hand Pose Estimation}

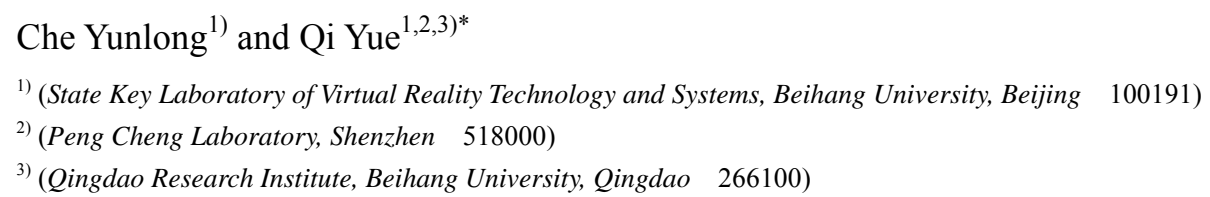

Abstract: Depth-based hand pose estimation has received increasing attention in the fields of human-computer interaction and virtual reality. A comprehensive survey and analysis of depth-based hand pose estimation of recent works are conducted. First, the definition and difficulties of this problem are explained, the widely used sensor and public datasets are also introduced. Then, the works of this field are divided into three categories, model-driven, data-driven, and hybrid method. The model-driven methods perform a model fitting between the model and the depth points. The data-driven methods learn a function, which maps the depth image to pose. The hybrid methods combine model-driven and data-driven to recovery the hand pose. In the course of narration, we focus on the solved problems and shortcomings to be solved. In the final, the works are compared in terms of accuracy, suitability, and robustness. The future research in this direction is also discussed.

Key words: depth image; hand pose estimation; hand tracking; deep learning; human computer interaction

基于视觉的裸手交互一直是人机交互、虚拟现 实和增强现实领域的研究热点，其应用十分广泛，
如在命令交互 ${ }^{[1-7]}$ 、手语识别 ${ }^{[8-10]}$ 和真实感抓取 ${ }^{[11-19]}$ 等多个方向中都需要准确地进行手部姿态估计.

收稿日期：2020-11-18; 修回日期：2021-02-28. 基金项目：国家自然科学基金(62072020); 广东省重点领域研发计划 (2019B010150001); 青岛市创新创业领军人才项目(19-3-2-21-zhc). 车云龙(1988一), 男, 博士研究生, 主要研究方向为计算机视觉; 齐越(1969-), 男, 博士, 博士生导师, 论文通讯作者, 主要研究方向为计算机图形学、三维重建、虚拟现实. 
现有的手部姿态估计方法可以分为有标记的 手部姿态估计和无标记的手部姿态估计. 前者主 要通过佩戴专用设备获取手部关节位置或关节旋 转角度, 如数据手套 ${ }^{[20-21]}$ 和彩色标记手套 ${ }^{[22-23]}$ 等, 或者在场景中布置复杂的多摄像头采集设备, 如 光学运动捕捉系统 ${ }^{[24]}$ 等. 从用户体验角度, 最理 想的是基于视觉的无标记方法, 即手部无须佩戴 任何设备就能准确进行手部姿态估计. 传统方法 主要是通过彩色光学相机采集手部运动图像, 再 运用计算机视觉相关算法 ${ }^{[25-26]}$ 估计手部姿态，其 准确性不高且鲁棒性很难达到实用需求. 其主要 原因是光学相机采集图像的过程中丢失了深度信 息，使从彩色图像恢复手部姿态面临二义性问题; 同时, 手运动速度较快且自由度高, 而不同人的手 部肤色多变，极大地增加了解决问题的难度; 而 且, 图像背景环境复杂, 图像质量容易受到环境光 的影响.

近些年，随着消费级深度摄像机的广泛使用， 利用深度图像估计手部姿态成为人机交互、虚拟现 实和增强现实领域的研究热点. Piumsomboon 等 ${ }^{[27]}$ 在增强现实环境中识别不同的手势, 可执行 40 个 不同交互任务. 2017 年, Krupka 等 ${ }^{[28]}$ 设计了基于手 部关键点的人机交互接口, 将手部关节点映射为手 部动作序列, 并利用这些动作序列执行相应操作. 在产业界，基于主动红外立体视觉的 Leap Motion 手部姿态追踪套件广泛应用于虚拟现实设备的人机 交互，能够以 180 帧/ $/ \mathrm{s}$ 的帧率追踪双手的运动. 2020 年, 微软发布了 HoloLensv2, 其内置深度相机能同 时捕获用户双手的姿态. 与彩色图像相比, 利用深 度图像进行手部姿态追踪有 2 个优势: (1) 深度相机 可以获取手部与相机之间的物理距离, 避免了求解 姿态时的二义性；(2) 深度相机成像质量不受室内 光照影响. 因此, 利用深度图像能够比较准确地把 手从复杂背景中分离出来. 然而, 采用深度传感器 也带来了另外的问题: (1) 目前深度传感器分辨率 低, 数据采集速度不高, 当手运动速度快时, 图像 会出现严重的运动模糊和边缘噪声; (2) 目前的深 度传感器工作场景侧重于室内, 且有效的工作范 围为 0.2 1.2 m; (3) 现阶段深度相机价格比彩色相 机高, 不利于推广使用.

基于深度图像的姿态估计方法主要有 3 类: (1) 基于模型驱动的估计方法 ${ }^{[29-35]}$, 通过进行三维手 部模型与输人的深度点云的配准以估计手部姿态; (2) 基于数据驱动的估计方法 ${ }^{[36-42]}$, 从大量的训练 样本中学习一个从输人深度图像到手部姿态的映
射函数; (3) 同时结合这 2 种方法的混合方法 ${ }^{[43-46]}$, 首先使用基于数据驱动的手部姿态估计方法训练 出姿态初始化器或寻找模型和点云之间的对应关 系, 然后使用基于模型驱动的手部姿态估计方法 迭代求解姿态.

本文首先定义基于深度图像的手部姿态估计 问题, 并论述手部姿态估计需要解决的难点; 接着 介绍主流的深度相机和常用的数据集, 然后论述 近些年出现的主要方法以及其主要解决的问题和 不足之处; 最后从多个角度对这些方法进行比较, 并展望未来工作方向.

\section{1 手部姿态估计的问题定义}

大多数文献将基于深度图像的手部姿态估计 的问题定义为从单幅或连续深度图像中恢复手部 关键点的位置(二维的图像坐标或三维的空间位 置)或手的自由度参数. 具体来说, 输人的是包含 手部的深度图像, 输出的是二维或三维手关节的 位置, 或是若干个自由度参数.

图 1a 所示为一个常用的 26 自由度手部动力学 骨骼模型, 其中不同的关节具有不同的自由度(根关 节点包换全局位移和旋转角度), 在一些方法 ${ }^{[47-48]}$ 中, 手部关节的长度也被认为是一种自由度. 图 $1 \mathrm{~b}$ 所示为骨骼模型经过前向动力学和线性混合蒙 皮后得到的手部几何面片模型. 具体而言, 在获得 各个关节的旋转角度后, 结合手的前向动力学和 给定的手部骨骼铰链结构可以重建出手的关节位 置和表面细节等信息. 另外, 手的自由度维度远小 于手的关节点坐标的维度, 一些方法 ${ }^{[49-50]}$ 利用该 特性来降低估计问题的复杂度.

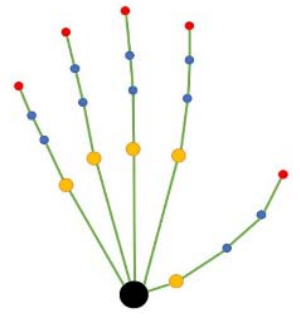

a. 手部骨骼模型

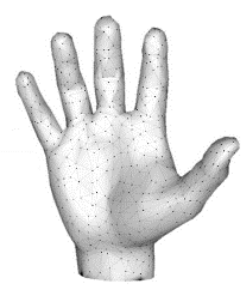

b. 手部面片模型
图 1 手部骨骼模型与其对应的面片模型

根据输入的深度图像是单幅深度图像还是连 续的深度图像序列, 可以将现有的方法分为 2 种: (1) 直接从单幅深度图像中恢复手部姿态; (2) 从 连续深度图像序列中估计出连续的手部姿态. 它 
们的区别在于，后者可以利用深度图像序列的时序 信息，该方法有时也被称为手部追踪(hand tracking).

根据深度相机和手的位置关系可以将现有的 方法分为 2 种: (1) 正向视角, 主要应用于基于姿 态控制的系统中, 在这种视角下手掌正对深度相 机, 手在图像中所占面积较小且手运动范围较大; (2) 自我视角, 主要用于虚拟现实和增强现实场 景, 在该视角下手距离深度相机较近, 手腕离摄像 机更近，手在深度图像中面积较大且自遮挡严重 (指尖经常被遮挡或移出图像之外). 图 2 所示为 2 种不同视角下的手的彩色图像和对应的深度图像.

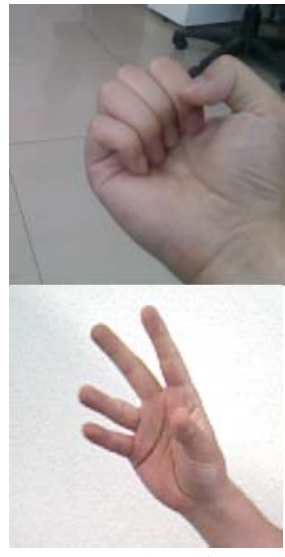

a. 彩色图像
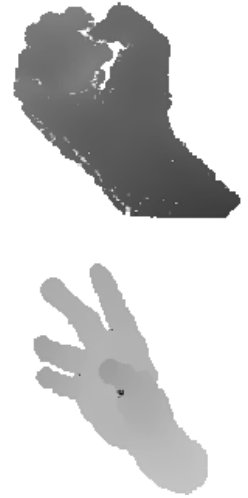

b. 深度图像
图 2 自我视角和正向视角下的手部图像

目前为止，基于深度图像的手部姿态估计仍 然是一个尚未被很好地解决的问题, 其主要面临 以下挑战.

（1）手的自相似性和自遮挡问题. 人的各个手 指的外形相似度较高, 这增加了辨识的难度. 手的 自由度较高, 铰链物体的高自由度带来的一个最 直接的问题就是手指之间的自遮挡严重，这些严 重的自遮挡可能导致手指的不同部分在图像中重 叠. 手部的自遮挡还包括 2 只手交互时相互之间发 生的遮挡. 这些情况有时也被称为不完整数据的 问题. 此外，在虚拟现实和增强现实场景中还面临 其他需要解决不完整数据问题. 例如, 手经常只有 一部分在视野中，或者经常移出视野或被其他物 体遮挡了一部分.

（2）深度相机硬件性能有限. 受深度传感相机 成像原理限制，现阶段的大多数深度相机在实时 采集速度 $(30$ 帧/s)情况下，最高分辨率一般为 480 像素, 部分双目视觉相机分辨率能够达到 720 像 素, 但是成像质量较差且帧率低(仅有 7 8 帧/s). 人的手具有快速移动能力, 手腕的移动速度甚至
可以达到 $5 \mathrm{~m} / \mathrm{s}$. 在采集速度仅有 30 帧 $/ \mathrm{s}$ 的情况下， 意味着前后 2 帧之间手的位置可能发生很大变化, 导致输出存在着空间上的不连贯现象(该现象也称 为抖动). 值得注意的是, 手的快速运动还会导致 深度图像产生严重的运动模糊和特有的边缘噪声, 这些复杂的噪声也增加了估计问题的难度. 当前 深度相机的工作距离比彩色光学相机小, 这也是 一个问题, 具体体现为常用的深度相机 (如 Intel RealSense)的有效范围仅为 $0.2 \sim 0.6 \mathrm{~m}$, 绝大多数深 度相机距离超过 $1.0 \mathrm{~m}$ 后获取的深度图像噪声严 重, 甚至无法识别手部轮廓. 另外, 深度相机的视 场相比光学相机狭窄.

\section{2 深度相机和公开数据集}

市场上深度相机按照成像原理可以分为飞行 时间方法、结构光技术或双目立体视觉技术. 基 于飞行时间方法的深度相机向场景发射脉冲或调 制后的光信号，再根据测量返回波的时间差估计 场景的深度信息. Foix 等 ${ }^{[51]}$ 对这些飞行时间方法 进行了总结. 基于结构光技术的相机向场景投射 预先设计好的红外点阵编码, 再使用红外摄像机 采集图像并比对采集到的图像和原有的红外点阵 得到场景的深度信息. Zanuttigh 等 ${ }^{[52]}$ 详细介绍了 结构光相机的工作原理. 立体视觉技术使用双目 立体视觉从光学图像中获取三维信息, 其原理类 似于人眼的成像过程，选择何种相机进行手部姿 态估计主要取决于所要解决的问题. Intel RealSense 的 F 系列和 Kinect $\mathrm{v} 2$ 适用于手部与相机之间 中等距离和远距离的应用, 而 Intel RealSense SR 系列和 Leap Motion 适用于手部与相机之间近距离 的场景.

计算机视觉领域中，数据集是不同算法准确 性比较的基础. 手部姿态估计的数据集可以运用 计算机图形学算法进行合成, 也可以使用深度相 机采集. 这些方法可以分为 2 类：(1) 使用计算机 图形学渲染合成方法; (2) 真实场景下数据采集后 离线标注方法. 前者使用计算机图形学算法将三 维手模型依照预设的相机参数投影到二维平面生 成合成深度数据, 其最大的优势是可以自由地选 择不同相机视角, 或者设定任意的手部姿势以覆 盖整个手部动作空间. 后者采集深度数据后使用 离线优化或人工手动标注数据, 本文根据采集所 使用的方式又分为可穿戴式采集、多相机同步采集 和单相机采集. 
三维手部建模工具(如 Libhand, Poser 等)已经 广泛应用于制作合成数据集. 由于基于渲染的方 法在生成深度图像的过程中已经隐式包含了标记 数据的过程, 因此采用图形学合成的方法生成的 标签信息是最准确的, 但是合成数据和真实图像 相比有明显差异，合成数据丢失了真实图像特有 的噪声, 而后期添加的一些噪声又比较简单, 无法 充分还原真实场景的特征.

一些方法在同时标定穿戴设备和深度相机后可 以获得大量深度图像和对应的高质量标注. $\mathrm{Xu}^{[53]}$ 等 利用数据手套标注并公开该数据集，其缺点在于 样本数量较少. Liang 等 ${ }^{[54]}$ 使用数据手套捕捉手部 动作的关节参数, 虽然使用数据手套可以直接获 得精确的关节标注, 但需要穿戴烦琐的硬件, 并且 场景内容单一, 获得的图像与真实数据仍有一定 的差距．还有一些方法采用单个或多个相机采集 数据并结合离线优化以提高标注的准确性. Tompson 等 ${ }^{[55]}$ 通过离线的粒子群算法 (particle swarm optimization, PSO)自动标注采集的深度数据，后续 为了提高准确性，使用人工手动调整这些自动标 注结果. 为了降低自遮挡的影响, 他们使用 3 个不 同视角的深度相机采集同一场景. 为了提高标记 的质量, Oberweger 等 ${ }^{[56]}$ 提出了一种数据集半自动 标注方法. 用户首先手动预先标记图像中可见关 节的二维坐标，作为方程的初始解，再通过优化损 失函数进行自动标记工作，在自动标记过程中利 用空间、时间和外观约束恢复整个连续数据帧上的 全部手部姿势.

表 1 所示为目前常用的数据集，其中“样本数 量”列表示数据集中样本的数目, “关键点”列表示 真值标签中关键点的数目. 本文将选取其中 4 个主 流使用数据集详细介绍.

(1) ICVL．该数据集于 2013 年由英国帝国大 学伦敦学院计算机视觉实验室发布, 包含 18 万幅 标注 16 个关节点的深度图像. 该数据集包含 10 个 主题，每个主题由不同的人录制，每个主题包含了 26 个不同姿势, 其最大的特点在于标注内容中包 含了手相对于相机的角度信息.

(2) NYU. 该数据集于 2014 年由纽约大学发 布, 训练数据集包含 72757 幅深度图像, 测试数据 集包含 8252 幅深度图像. 这些深度图像来自同一 真实场景 $(2$ 个人录制)，由 3 个不同视角的深度相 机拍摄，每幅深度图像有 36 个关节点的三维标注.

(3) MSRA15. 该数据集于 2015 年由微软亚洲 研究院孙剑等发布，包含 76375 幅深度图像，每幅
表 1 常用的公开数据集

\begin{tabular}{lcrc}
\hline \multicolumn{1}{c}{ 数据集 } & 发表年 & 样本数量 & 关键点 \\
\hline ASTAR $^{[54]}$ & 2015 & 870 & 20 \\
Dexter1 $^{[57]}$ & 2013 & 2137 & 6 \\
UCI-EGO $^{[58]}$ & 2014 & 400 & 26 \\
MSRA14 $^{[59]}$ & 2014 & 2400 & 21 \\
NYU $^{[55]}$ & 2014 & 80000 & 36 \\
ICVL $^{[60]}$ & 2013 & 332500 & 21 \\
MSRA15 $^{[61]}$ & 2015 & 76375 & 21 \\
FingerPaint $^{[62]}$ & 2015 & 100000 & 21 \\
HandNet $^{[63]}$ & 2015 & 212000 & 6 \\
Dexter+Object $^{[64]}$ & 2016 & 3014 & 5 \\
STB $^{[65]}$ & 2017 & 18000 & 21 \\
RHD $^{[66]}$ & 2017 & 43000 & 21 \\
SynthHands $^{[67]}$ & 2018 & 63530 & 21 \\
BigHand2.2M $^{[68]}$ & 2017 & 22000000 & 21 \\
FHAD $^{[69]}$ & 2018 & 100000 & 21 \\
\hline
\end{tabular}

图像有 21 个关节点标注信息. 这些图像分为 9 个 主题，每个主题包含 17 种不同手势. 该数据集的 最大特点是手的姿态空间分布比较平均.

(4) BigHand 2.2M. 它是至今为止手部姿态估 计方向数量最多的数据集, 2017 年由英国帝国大 学伦敦学院发布. 其包含 200 多万幅标记 21 个关 节点的手部深度图像, 用 Kinematic $6 \mathrm{D}$ 摄像机采 集. 数据集分为 10 个主题, 每个主题采集不同人 的手部数据.

现阶段的研究者主要使用 ICVL，MSRA15 和 NYU 这 3 个数据集进行验证实验，因为它们的样 本数量多, 且样本在手的姿态空间采样均匀, 标签 标注质量较高. 因此, 本文使用这 3 个作为基准数 据集，比较目前常用方法的准确性.

\section{3 主流工作}

对于一个完整的手部追踪系统, 手部检测和 手部姿态估计密不可分. 因此, 在论述现有手部姿 态估计方法前，首先对现有方法中使用的手部检 测方法进行概述。

大多基于深度图像手部姿态估计方法都默认 手部区域已经检测并分割出来. 这些方法大多数通 过基于最近物体阈值的原理实现手部定位，即根据 深度距离阈值分割出手和背景. 还有一些基于颜色 的方法，如文献[70-71]使用了基于腕带的手部检测 方法(图 3a), 检测的手腕方向作为额外的约束项(通 过手腕的朝向可以额外计算出手的旋转信息)嵌人 
姿态优化方程中. Choi 等[72]使用卷积神经网络同时 进行手的检测和分割, 直接使用整个深度图像作为 输人, 同时输出手部检测框、手的中心位置和手部 语义分割. Oikonomidis 等 ${ }^{[73]}$ 用基于肤色的手部检测 算法分割手部区域, 图 3b 所示为基于肤色的手部 区域分割结果. 还有一些方法 ${ }^{[74-75]}$ 使用人体关节点 跟踪器 ${ }^{[76]}$ 估计手的粗略位置. Taylor 等 ${ }^{[77]}$ 将手部检 测视为一个手和背景的二值分类问题, 其分类结 果如图 3c 所示. 另外, 还出现了使用卷积神经网 络(convolutional neural networks, CNN)进行手部检 测的方法.

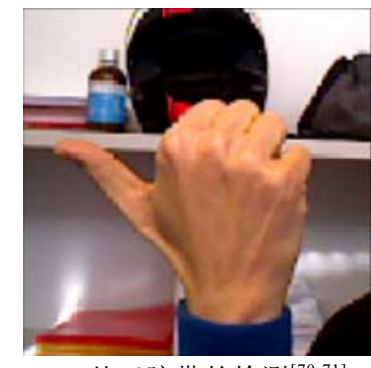

a. 基于腕带的检测

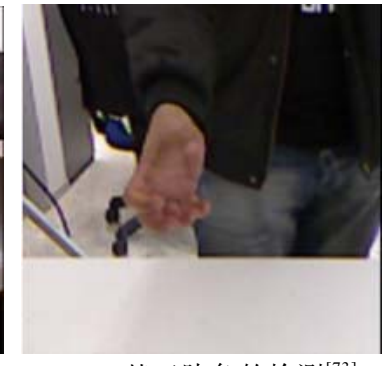

b. 基于肤色的检测 ${ }^{[73]}$

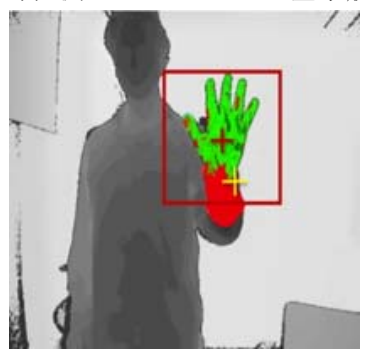

c. 基于像素分类 ${ }^{[77]}$

图 3 几种常见手部检测方法

\section{1 基于模型驱动的方法}

基于模型驱动的方法将手部姿态估计转化为 点云配准的优化问题, 其试图最小化点云和一个 预先设定好的参数化手部模型间的距离残差. 由 于该问题高度非凸且手部自由度维度高，遍历整 个搜索空间寻找最优解往往无法满足实时需求. 从理论上讲，现有方法不管采用全局最优还是局 部最优, 本质上都是寻找某个姿态使目标函数在 某处的残差最小.

现有模型驱动方法对优化方程的初始值非常 敏感. 因此, 很多方法采用启发式的姿态初始化和 局部搜索相结合的方法求解次优解, 有的方法 ${ }^{[71]}$ 默认手一开始就处于屏幕中心, 这也常被认为一 种隐式的初始化方法. 另外, 一些方法 ${ }^{[73-74]}$ 用前一 帧的结果作为下一帧的初始值, 这种方法往往由 于累积的估计误差而极容易陷人追踪失败，而手 的高自由度导致模型驱动方法很难从追踪失败中
恢复，这也是基于模型驱动的方法面临的最大问 题. 最近的一些工作在采用了基于模型驱动方法 的同时结合了深度学习, 这些方法归纳于混合驱 动方法并在后续章节进行介绍. 在介绍相关工作 前, 首先对模型驱动方法涉及的一些关键技术进 行介绍.

手的前向动力学(forward kinematic, FK)描述 了如何将手部自由度参数映射为手的骨骼模型和 几何面片模型. 给定手部自由度向量和预定义的 手层次骨骼模型, 根据前向动力学可以计算出手 各个关节点位置(即骨骼模型), 再结合线性蒙皮算 法, 可以进一步获得手部的几何网格模型. 与此相 反，从手部关节点位置计算出手部自由度的方法 称为逆向动力学( inverse kinematic, IK), 其常用于 模型驱动的离线优化过程中.

PSO 是一种全局优化算法, 其通过在若干迭 代次数内进化若干粒子实现寻找最优解. PSO 非常 适合于求解非光滑目标函数, 常用于非凸问题寻 找全局最优解. 早期模型驱动方法 ${ }^{[73,78]}$ 基本都采 用 PSO 估计手部姿态.

最近点迭代(iterative closest point, ICP)算法常 用于三维点云配准问题. Fleishman 等 ${ }^{[79]}$ 提出了结 合逆向运动学的 ICP 算法. Qian 等 ${ }^{[59]}$ 发现, ICP 能 够快速达到局部最优，而 PSO 能更有效地探索整 个参数空间，但存在过早收玫的问题，因此他们提 出了 ICP-PSO 方法, 即将 ICP 和 PSO 结合并搜索 整个参数空间, 使其迅速收玫到某个局部区域得 到最优解，其在单个 CPU 上的速度达到 24 帧/s.

对于同样是采用 ICP 的基于模型驱动的方法, 根据每次迭代时计算梯度雅可比矩阵的区别，可 以分为数值求解方法和解析求解方法. 由于手的 自由度参数较多, 因此手的前向过程计算复杂高, 进而导致前者的计算复杂度远高于后者 ${ }^{[79]}$, 这也 是 PSO 方法远慢于梯度下降法的主要原因之一.

$\mathrm{Tkach}$ 等 ${ }^{[70]}$ 提出了基于 ICP 的手部姿态优化方 法，其使用经典的“渲染-计算”,[62]框架，并公开了 其代码, 对后续工作影响很大. 该方法使用深度和 彩色图像作为输人, 并使用圆柱体表示手部模型, 进行优化求解. 这个过程使用 ICP 获得三维点云和 模型对应关系，利用距离变换算法提取二维轮廓 生成像素-模型对应关系，使用梯度下降算法迭代 计算进行优化，其中使用了手部关节旋转约束、时 序约束、手部运动空间约束和关节碰撞约束等. 但 该框架最大的瓶颈是，在模型绘制时上下文的切 换耗时很大. 
除了传统的基于几何优化的模型驱动方法, 还有一些方法试图引人刚体动力学 ${ }^{[80-81]}$ 、高斯混合 模型 ${ }^{[82-83]}$ 估计手部姿态. Melax 等 ${ }^{[80]}$ 采用基于物理 模拟的方法对手部模型和深度点云的配准，使用 17 个圆柱刚体模型表示手, 通过求解刚体动力学 方程解决手部姿态估计问题. 在求解过程中同时 引人刚体碰撞检测等手部先验约束, 并在数据约 束项中使用基于点到平面的三维数据约束, 使收 玫过程更加平滑. Sridhar 等 ${ }^{[82]}$ 用高斯模型表示手 (即在手的关节等处用高斯模型代替手部几何面片 模型), 将输人的点云和手部都表示为高斯分布, 最后用局部梯度下降的方法优化手部姿态. 该算 法在保持较高精度的同时, 仅需要 CPU 硬件的支 持即可达到实时效果.

大多数基于模型驱动的方法采用固定大小的 三维手模型匹配点云，但不同人的手大小不同且 关节长度比例也不同. 手的自由度信息除了包含 各关节的旋转角，还应该包含各关节之间的距离， 用固定尺寸的三维手部模型进行优化, 会降低算 法的准确性. 因此, 在优化过程中, 考虑将手的长 度作为变量很有必要. Khamis 等 ${ }^{[84]}$ 在优化过程中 将手部骨骼的长度作为变量加人优化过程, 只需 要使用 15 幅连续深度图像就能完成离线手部形状 的标定. Remelli 等 ${ }^{[48]}$ 提出了离线手部标定算法, 使用一个球体模型表示三维手部模型, 将手的形 状作为变量加人优化过程中进行全自由度优化(即 同时优化手的姿态和形状), 在优化目标方程中同 时引人了点云-模型的误差和模型-点云的误差. 图 4 所示为其多阶段优化的过程.
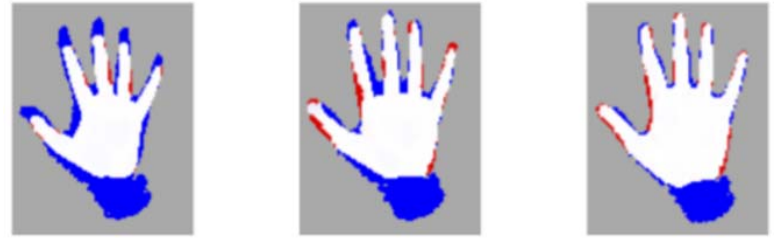

图 4 Remelli 等 ${ }^{[48]}$ 联合手部形状进行优化的示意图

模型驱动的方法极度依赖于初始解的选择和 优化方程的目标函数设计, 求解得到的姿态往往 具有空间上的连贯性且符合物理自然约束. 因此, 近些年来基于模型驱动的方法侧重于研究不同的 手部模型表示(如采用可变尺寸的手部模型或探讨 如何在模型精细化和计算速度之间均衡), 或者采 用不同的先验约束项确保求解得到的手部姿态的 结果更为真实. 但是, 由于消费级深度相机采集的
深度图像质量较差, 模型到点云的配准过程中易 产生误差累积使追踪失败. 仅仅依赖一些启发式 的方法很难从追踪失败中恢复出来，这也是基于 模型驱动的方法最大的缺点.

\section{2 基于数据驱动的方法}

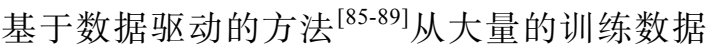
中学习一个从深度图像到手部姿态的映射函数. 与基于模型驱动的方法不同, 基于数据驱动的方 法用带标记的手部深度图像训练分类器或回归模 型. 这些经过训练后的分类器或回归模型, 可以从 输人的深度图像序列中恢复出对应的手部姿态, 直接处理单幅深度图像, 不需要基于模型驱动方 法的初始化过程, 因而追踪过程不存在误差累积. 例如, 手从相机视图中消失后再次进人, 系统能继 续运行. 基于数据驱动的方法的最显著的特点是 必须依赖足够多的训练样本才能实现较好的泛化 能力, 这里的“足够多”是指抽样的手部姿态能够 均匀地覆盖整个手部运动空间. 下面按照所用的 分类器/回归模型种类, 论述一些典型的基于数据 驱动的方法.

Keskin 等 ${ }^{[10]}$ 延续了 Kinect 人体骨架关节点追 踪的“逐像素部件分割+区域聚类中心”的模式，对 输人的手部图像使用训练过的随机森林(random forest, RF)进行逐像素部件归属分类, 并使用均值 漂移算法寻找类的中心，然后将寻找到的位置向 $Z$ 方向平移, 获得最终的关节点. 该方法对于某些极 端视角下的输人图像的泛化能力较差. Tang 等 ${ }^{[87]}$ 使用隐变量回归方法, 直接从深度图像中估计手 部姿态, 其根据手的拓扑结构由粗到细层级地寻 找隐节点对应拓扑结构的重心位置, 最终找到手 的各个节点位置.

Sun 等 ${ }^{[61]}$ 将传统的二维图像的级联姿态回归 拓展到三维手部姿态估计上, 优化了以往基于 $\mathrm{RF}$ 方法使用的二维像素差异特征，同时针对手的特 殊拓扑结构提出了三维层次级联回归的方法. 具 体做法如下：(1) 对手进行归一化以增加旋转不变 性; (2) 对归一化后的手部关节位置提取三维姿态 索引特征；(3) 基于这些三维姿态索引特征进行层 级级联回归. 与之前的级联算法不同的是, 该算法 先估计手掌的位置, 然后分别估计各个手指的位 置. 这是因为手掌的位置相对于手指更加稳定, 而 且手掌位置的变化更会显著地影响手指的估计, 且手指之间的估计是互相独立的. Sun 等 ${ }^{[61]}$ 分别在 ICVL 和 MSRA15 这 2 个数据集上进行了验证, 由 于采用了层级学习残差和手掌/手指分开回归的策 
略, 与隐变量回归 RF 相比, 其在指尖的估计精度 上有提高. 该工作是目前基于 $\mathrm{RF}$ 方法中准确度和 速度最高的．基于 $\mathrm{RF}$ 的方法大多集中于 2015 年以 前, 根据其公开结果, 这些方法都能够满足实时的 追踪需求. 但是由于在这些方法中选取的都是局 部梯度特征, 该特征只能描述某个点附近的三维 局部纹理信息，而深度图像的噪声会随着手的运 动速度剧烈增加. 如果输人的图像噪声过大, 会导 致 RF 方法的准确性迅速下降.

$\mathrm{CNN}$ 相对传统机器学习方法在准确性和鲁棒 性等方面有很大优势. Tompson 等 ${ }^{[55]}$ 用多分辨率输 人的 CNN 将原图根据平均深度归一化, 分别降采 样到 3 种分辨率，再分别输人网络生成的关节点分 布热图. 这种多分辨率输人能够使其网络分别学 习到全局和局部特征，同时，该策略也对后续的很 多工作具有借鉴意义.

2015 年开始, 出现了大量了基于 $\mathrm{CNN}$ 手部姿 态估计的方法 ${ }^{[86-87]}$, 这些工作采用不同的网络结 构提取手部特征. Oberweger 等 ${ }^{[88]}$ 用 CNN 回归手部 姿态, 其使用多尺寸和多阶段的 CNN 直接做姿态 的回归，第 1 阶段使用网络回归生成粗略的手部关 节位置，第 2 阶段回归生成第 1 阶段关节位置和样 本真值之间的残差(即采用多阶段级联的策略). 该 方法的一个贡献在于提出基于主成分分析的姿态 先验层，并基于此提高了预测精度，但实际上普遍 认为这是隐式地进行参数降维, 并没有真正利用 手的先验知识.

有些方法尝试将手的先验知识显式地加人神 经网络中. Zhou 等 ${ }^{[50]}$ 将手的前向过程嵌人 CNN 中, 提出了一个无参数的手模型层 (hand model layer), 该层将手部自由度通过可微的前向动力学函数映 射为手部关节点坐标, 同时使用关节旋转的约束 作为新增的损失函数项; 但是由于其选取的特征 提取网络比较简陃，因此准确度比同时期其他工 作提升有限.

除了传统的二维图像作为神经网络的输人外, 还有尝试采用其他方式表示深度图像的工作 ${ }^{[89-91]}$. $\mathrm{Ge}$ 等 ${ }^{[89]}$ 首先将输人的深度图像重新投影到 $Y$ 平面 和 $Z$ 平面上，通过多个卷积层各自生成对应的热力 图, 然后进行多视角融合输出最终的关节位置. 与 传统的二维图像相比, 这些三维表示能够更好地 描述深度图像的特征，并学习到多个视角下的点 云特征。

除了采用不同类型的图像输人外，使用基于 无序点云的网络结构也是一大研究热点. $\mathrm{Ge}$ 等 ${ }^{[00]}$
用基于点云的网络进行手部估计，比传统 CNN 提 高了准确性. 其直接使用 PointNet 作为特征提取 模块进行姿态估计, 一个创新点在于提出基于包 围盒的数据增强方式和指尖增强网络用于提高指 尖关节的预测精度, 这种增强网络本质上是一种 级联式的学习方式. 其提出的基于包围盒的数据 增强方式能在一定程度上解决深度点云噪声过大 的问题. Chang 等 ${ }^{[22]}$ 使用基于“三维体素输人-三维 体素热图输出”的方式进行手部姿态估计, 其网络 使用体素化的深度点云作为输人, 逐体素地对手 部关节点位置进行最大似然估计. 值得一提的是, 这篇文章同时获得了 The 2017 Hands in the Million Challenge on 3D Hand Pose Estimation 的冠军.

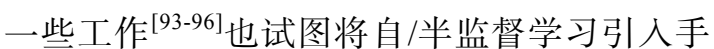
部姿态估计中. Wan 等 ${ }^{[96]}$ 提出了一个自监督的框架 解决手部态中训练样本难以获取的问题. 其输人 图像首先经过一个姿态估计网络(在合成数据上训 练而成)输出手的关节位置, 并通过这些关节位置 生成三维的手部球体模型并投影到指定平面上, 然后使用这个投影出来的合成深度图像和输人的 深度图像进行模型匹配，并将其作为整个网络的 损失. 该方法能够很好地解决现阶段关于手的训 练样本不够的问题.

2018 年后, 已经没有太多针对单手的基于深 度图像的姿态估计工作出现在三大计算机视觉顶 会, 研究热点开始转移到估计抓取物体时的手部 姿态, 其中具有代表性的工作是 Doosti 等 ${ }^{[97]}$ 提出 的 HOPE-Net，该工作通过训练过的神经网络同时 估计手部姿态和物体的姿态.

近年来，基于数据驱动的方法成为整个领域 的主流，相关研究也大多集中在这个方向，而它们 在公开数据集上的表现也证明了其准确度比基于 模型驱动的方法有了很大的提升.

根据网络输出方式的不同可以将这些工作分 为检测方法和回归方法, 前者使用关键点热图回 归的方式恢复出手的关键点位置的最大似然概率, 后者则直接通过全连接层输出归一化后的关键点 坐标. 前者的准确度取决于热图的分辨率，由于逐 像素的计算耗时较多, 后者一般在特征提取网络 后直接用全连接层输出关键点位置信息，缺点在 于准确度不如检测方法. 从网络的输人角度可以 分为使用二维深度图像、三维体素、无序点云和图 卷积等. 一系列工作也证明了与单纯的二维网络 相比, 三维数据结构能够更好地表示手部的几何 信息. 
同时，基于数据驱动的方法也仍然面临着很 多问题. 首先, 大多数基于数据驱动的方法只使用 单幅图像作为输人, 无法有效地利用时序信息, 预 测的结果存在着空间上的不连贯性. 其次, 这些网 络模型并没有真正意义上利用手的各种先验信息， 使其预测的结果往往不符合物理约束. 这些方法 还有一个最明显的问题在于, 其网络对于输人数 据非常敏感, 而剧烈运动导致的大量噪声可能降 低整个网络模型的准确度.

\section{3 混合方法}

基于模型驱动的方法和基于数据驱动的方法 在许多方面存在互补性. 基于模型驱动的方法需 要姿态初始化才能进行追踪, 而基于数据驱动的 方法往往无法利用手的先验信息而使结果缺乏空 间连贯性. 因此，一些工作提出的混合方法将上述 2 种方法结合在一起，用于解决手部姿态估计问 题. 其使用一个训练过的分类器或回归模型对手 部姿态进行初始化, 这里的初始化的用途可以是 寻找逐像素的点云-模型的对应关系，也可以是利 用数据驱动的方法估计优化方程的初始近似姿态 值. 通过初始化得到方程初始值或语义上的点云模型对应关系的优化方程, 能够更快速、准确地求 解最终结果.

Krejov 等 ${ }^{[98]}$ 将基于 RF 逐像素分割的方法和基 于物理的模型拟合的方法相结合，先利用 RF 进行 手的区域分割, 再利用基于刚体动力学的方法进 行优化，这种混合策略能从由复杂视角导致的手 部姿态丢失的情况下迅速恢复. Sridhar 等 ${ }^{[83]}$ 也采 用这种“区域分割+模型拟合”的策略进行手部姿态 估计，但是其使用了高斯混合模型代替前者基于 物理的优化方法.

Sharp 等 ${ }^{[62]}$ 提出了一个实用的手部追踪系统, 声称其具有准确(对不同手势都有准确的估计结 果)、鲁棒(使用初始化器, 使系统能从各种追踪失 败中迅速恢复)和自由(该系统只需要一个 Kinect v2 摄像头且对于手的位置和角度没有特殊限制)3 大特点. 该系统包含了重初始化模块和模型拟合 模块. 重初始化模块使用了 2 层分类器的方法, 第 1 层用于预计手的全局旋转, 第 2 层用于预测具体 的手部姿态. 与之前使用 $\mathrm{RF}$ 直接回归手部姿态不 同, 这里的第 2 层分类器只是产生姿态的分布，以 进行一系列不同的姿态的估计. 模型拟合模块将 粒子算法和基因演化算法相结合，通过定义黄金 能量函数为对应位置深度的截断距离的和生成多
个姿态，使用基因算法的策略对于一些能量比较 高的粒子进行随机变换，最终经过多次迭代，并选 取最低能量的粒子所代表的姿态作为最终结果.

Taylor 等 ${ }^{[77]}$ 延续了渲染-计算 ${ }^{[62]}$ 的框架，但是 使用一个手势索引 $\mathrm{RF}$ 代替了原来的 2 层手部姿态 初始化 RF，又用基于梯度下降的优化方程代替了 原有的基于 PSO 和基因演化算法的模型匹配. 其 中, 使用融合了时序信息和手部姿态检索 RF 的输 出作为当前的初始化姿态, 从这个初始化姿态出 发, 结合多种手部先验约束(如碰撞、时序、关节 旋转等)进行手部姿态优化. 在后续工作中, Taylor 等 ${ }^{[99]}$ 将该算法拓展到交叉的双手上，使用一个手 部分割网络 ${ }^{[100]}$ 区分出左右手的像素归属，再用基 于梯度下降的方法优化手部姿态. 在优化过程中 借鉴了现有的 SLAM $^{[101]}$ 系统中的多种思路, 如用 卡尔曼滤波平滑前后帧的姿态.

混合方法在某种意义上结合基于数据驱动的 方法和基于模型驱动的方法的优势，使用数据驱 动作为姿态初始化工具可以很快地从追踪失败中 恢复, 而使用优化方程进行点云-模型配准可以使 输出结果在满足空间上的连贯性的同时, 保持物 理约束. 当遇到一些噪声过大的图像时, 能够使用 时序信息和运动空间约束平滑当前的结果. 这种 组合多种方法的策略在一定意义上使混合方法的 输出结果非常平滑, 这对于整个人机交互系统非 常重要, 用户在使用裸手进行交互时不会感觉到 迟滞感, 也能够及时地体会到视觉反馈.

\section{4 总结和展望}

下面对主流的手部姿态估计方法中的一些典 型工作的准确性、适用性和鲁棒性等进行评估.

\section{1 准确度}

定义一个合理的评估指标对于比较算法的准 确性至关重要. 现有的手部姿态估计算法主要采 用 2 个流行的评估指标: (1) 预测到的关节点与真 实结果之间的平均欧几里得度量；(2) 所有预测的 关节位置和真实值之间距离低于某个國值占总体 的比例. 此外, 一些试图恢复手模型的方法采用手 部模型和三维点云之间的误差作为评价指标用于 衡量算法的准确性，但是该指标仅局限于基于模 型驱动的方法.

本文主要采用主流的三维平均关节误差和误 差距离阈值占比这 2 个评价指标, 图 5 和图 6 所示 为在 2 个常用的公开数据集上 (ICVL, MSRA) 的一 
些代表性工作的比较结果 ${ }^{\circledR}$.

从图 5 和图 6 可以看出, 随着新的网络结构和 数据表示不断地被应用于手部姿态估计, 在各个 数据集上的最先进工作(state of the art, SOTA)的准 确度不断提高. 总体可以看出：(1) 基于深度学习 的算法比传统的机器学习 (这里主要是以 $R F$ 为代 表的工作)更准确; (2) 更复杂的网络结构和更高效 的数据表示确实能更好地提升模型的预测精度; (3) 训练数据集的数量提升能够更好地提高模型 的泛化能力.

对于深度图像的数据集, 训练集和测试集的 数据在某些方面上差距没有传统彩色数据集那么 明显, 使得基于数据驱动的方法比基于模型驱动 的方法在测试集上的表现更准确. 然而, 该准确性 仅仅是在单幅图像上取得的结果. 因为现有的深 度相机往往只能保证 30 帧 $/ \mathrm{s}$, 所以基于数据驱动 的方法追踪效果较差(其结果存在严重的抖动或空 间上的漂移).

基于模型驱动的方法和混合方法虽然在数据 集上的准确度没有基于数据驱动的方法高, 但是其
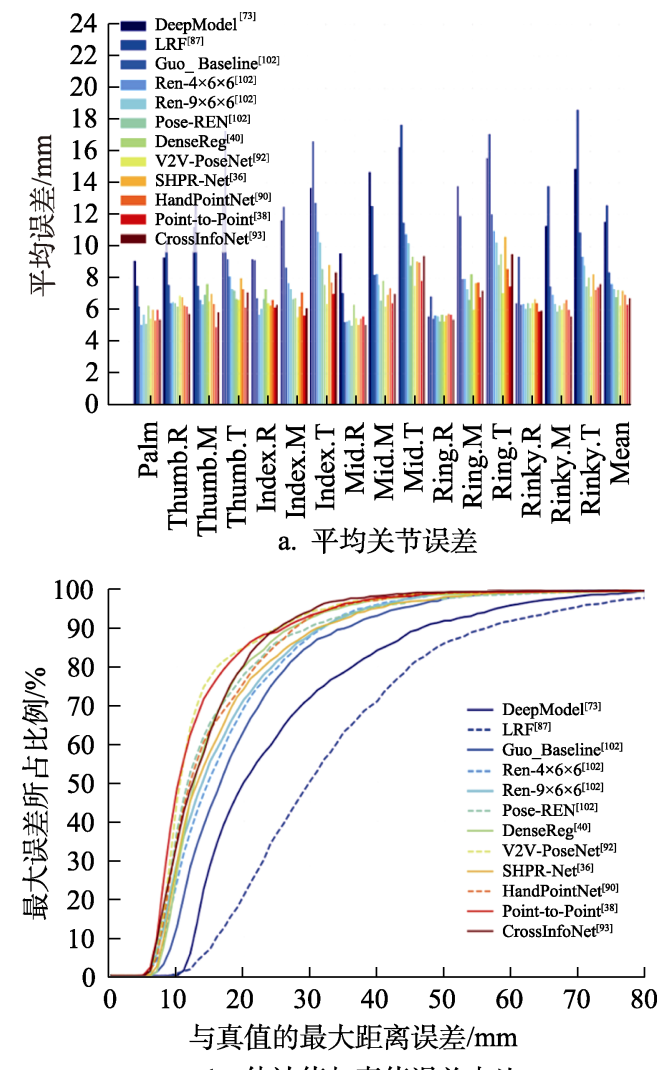

b. 估计值与真值误差占比

图 5 ICVL 数据集上不同算法的比较结果

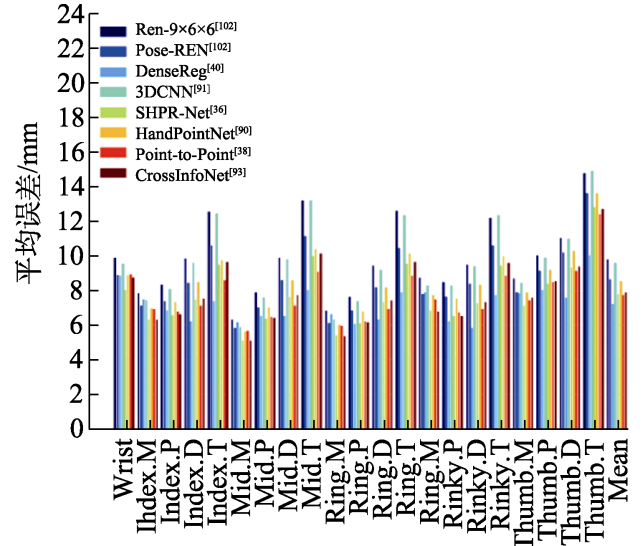

a. 平均关节误差

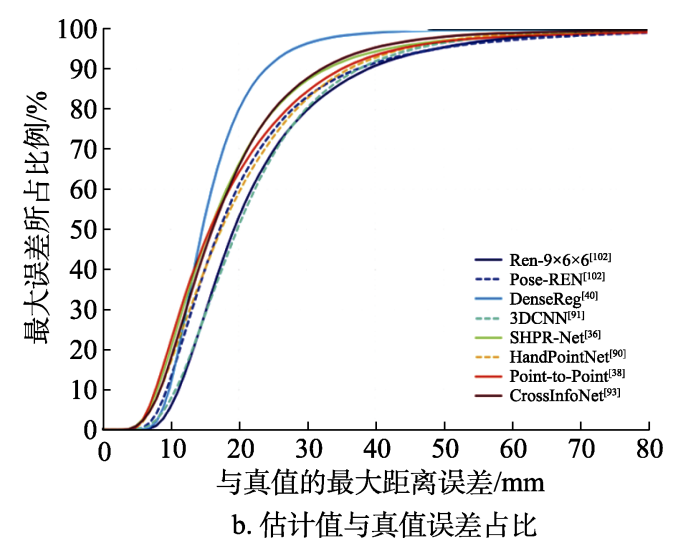

图 6 MSRA 数据集上不同算法的比较结果

能够充分利用手的时序信息和物理学约束, 使获 取的追踪结果更加真实. 换言之, 其追踪到的结果 更加平滑, 更符合人机交互系统的要求. 一些结合 了时序先验约束的函数项被用于平滑前后帧的结 果. 例如, Taylor 等 ${ }^{[77]}$ 通过在能量函数中加人时间 先验项，不仅保证前后帧手部姿势的时间一致性， 而且使得追踪算法的输出更为平滑; Tkach 等 ${ }^{[70]}$ 使 用前后帧能量项和手部运动空间能量项平滑前后 帧的结果, 也起到了平滑输出的结果.

\section{2 适用性}

尽管绝大多数模型驱动的方法都能满足实时 性, 然而它们对硬件均存在着一定需求. Qian 等 ${ }^{[59]}$ 提出的结合了 ICP-PSO 的方法在单核 CPU 上可以 到达 25 帧/s; Sridhar 等 ${ }^{[83]}$ 的高斯混合模型也在 $\mathrm{CPU}$ 上取得了 60 帧/ $\mathrm{s}$ 的结果; 而采用了更加精细 手部模型的一些手部建模/追踪算法, 需要昂贵的 GPU 才能满足实时性的要求. 这类算法由于手部 参数的高维度特征和复杂的手部前向动力学, 其中 基于 PSO 的群体算法都显著慢于基于 ICP 的算法. 此外, 基于简化手部模型的算法也都明显快于那

(1) https://github.com/xinghaochen/awesome-hand-pose-estimation 
些基于精确手部模型的算法.

绝大多数基于数据驱动的方法采用传统的机 器学习模型(如 RF), 只需要依赖 CPU 就能够满足 实时性的要求, 其声称能达到 300 帧/s. 而尽管近 年来依赖于 CNN 的方法在准确性和鲁棒性上有了 提升, 但是其网络都严重依赖 GPU, 且大多数网 络无法在 CPU 上达到实时的效果. 一些混合方法 的混合策略虽然在整体上提升了追踪的准确度和 鲁棒性，但是它们大多运行速度都低于 50 帧/s. 本 质上混合方法是基于数据驱动的方法和基于模型 驱动的方法的结合, 其运行一个姿态初始化模型 再加上模型优化的消耗明显大于单独的基于数据 驱动的方法和基于模型驱动的方法; 有时, 快速的 手部运动会导致频繁地调用重初始化模块, 这意 味着追踪失败, 需要运行姿态初始化模型, 从而进 一步降低了整个混合方法的速度.

整体上，手部追踪模块只是属于整个人机交 互系统的一部分，手部追踪占据的计算资源应该 尽量少. 在已经发布的 HoloLens v2 中, 手部追踪 算法已经被嵌人专用的芯片中. 可以看出, 未来的 趋势是在保证追踪算法准确性和鲁棒性的前提下， 使算法消耗的计算资源更少. 因此，在设计手部姿 态估计算法时，必须要考虑算法的部署成本问题.

\section{3 鲁棒性}

人机交互系统的鲁棒性对于用户体验至关重 要, 一般认为手部姿态估计算法的鲁棒性包含了 多个层面。

（1）追踪过程的稳定性. 手部追踪算法是否能 够在追踪过程中一直保持追踪状态? 如果追踪失 败, 能否快速地从失败中恢复? 一般认为, 混合方 法的鲁棒性比基于模型驱动的方法和基于数据驱 动的方法较高. 这归功于姿态初始化器和优化方 程的同时使用. 混合方法能很快地从追踪失败中 恢复，同时保持较高精度的追踪效果. 而仅仅使用 基于模型驱动的方法很容易在手部的快速运动中 陷人目标丢失的情况. 基于数据驱动的方法无法 较好地利用时间序列先验约束等, 其追踪结果易 出现抖动现象且无法感知当前的追踪状态.

(2) 输人的鲁棒性. 切换数据源是否需要将现 有的算法重新适配? 在飞行时间相机下运行良好 的算法是否能够直接移植到结构光相机中? 基于 模型驱动的方法往往对不同的数据源的输人泛化 较好, 因为基于模型驱动的方法本质上是求解点 云配准问题, 而简单地替换输人数据的类型(更换 后依旧是点云, 改变的可能是点云的稠密程度)并
不会较大改变配准的结果. 对于数据驱动的方法, 手部姿态估计的结果在很大程度上依赖带标注的 训练样本, 而不同的深度相机获取的深度图像千 差万别, 因此, 其对不同类型的输人数据较为敏 感, 泛化能力较差.

(3) 交互过程的自由. 追踪算法是否能够追踪 不同尺寸、不同距离和不同相机角度下用户的手? 为了提高用户交互过程中的自由度，基于数据驱 动的方法往往只能依赖增加不同距离、不同视角和 不同尺寸的数据, 但这样也显著地增加了训练成 本. 而受现有深度相机性能的约束, 现有深度数据 的有效范围较小, 大多数深度相机的有效距离范 围往往不超过 $0.6 \mathrm{~m}$, 在这个狭窄的视场内, 据现 有公开的展示结果, 混合方法在使用体验上比前 两者更为出色, 主要归功于初始化器和模型优化 的结合.

\section{5 结 语}

近年来, 无论是在学术研究还是商业应用中, 基于深度图像的手部姿态估计的算法都取得了令 人瞩目的进展, 其在公开的基准数据集上的最佳 纪录不断被打破. 一些方法也不断地尝试解决现 有深度设备的限制、手的自相似性和遮挡等问题. 但是本文认为以下关键问题仍然具有一定的研究 价值.

（1）现有深度相机的限制. 不管是手的快速运 动还是手与摄像机的距离过远, 都会导致严重的 深度噪声, 这些噪声包括运动模糊和边缘轮廓缺 失. 现有方法并没有真正去除这些噪声, 使得基于 深度学习的方法对于不同类型的深度图像泛化很 差, 在某个数据集中准确度很高的模型往往在另 外一个数据集中表现很差. 为了提高神经网络对 于输人图像的鲁棒性, 考虑在数据增强的方面使 用图像旋转、缩放以及添加运动模型等方法或使用 非图像的数据表示类型, 如使用 PointNet 或图网 络表示点云数据. 现有的基于模型驱动的方法对 输人较为鲁棒, 其需要解决的主要问题在于, 边缘 噪声某些情况下会造成估计结果在空间上的抖动, 一般会利用时序约束和运动空间约束平滑当前的 估计结果和前一帧的输出. 因此, 对于基于模型驱 动的方法, 探讨更有效的运动空间/时序约束的表 示仍十分必要.

(2) 手的自相似和自遮挡. 现有的深度学习的 方法在各种数据集上准确度的不断提升, 证明了 
复杂的网络结构和高效的数据表示能够有效地学 习手的各种几何信息, 这些训练过的网络能够区 分手的各个部件, 也能从不完整的数据中恢复手 部姿态. 然而现有的基于深度学习方法还有众多 问题需要解决. 首先, 尽管各种复杂的网络架构已 经被应用于解决此问题，但是随着神经网络结构 搜索(neural architecture search, NAS)技术的发展， 寻找更有效的网络架构仍存在着发展的空间. 其 次，现有的网络对时序信息以及手的各种先验信 息的利用不充分，导致输出的结果往往不符合物 理学约束, 因此在网络结构中有效地嵌人手的各 种先验对于提高预测结果的准确性有重大意义. 对于遮挡这个问题，现有方法都只解决一只手的 手指之间的自遮挡，然而对于 2 只交互在一起的 手，只有一些混合方法延续先分割后配准的思路 进行解决, 鲜有别的类型的方法进行尝试. 最后, 深度学习需要大量的训练数据, 而标记的难度增 加了训练的成本，因此探讨无/半监督的方法也很 有意义.

除此之外, 还有一些问题也有研究的价值. 例 如, 现有研究主要集中在室内的单一场景, 室外场 景可能受到复杂光照和动态背景的影响，现有的 TOF(time of flight)和结构光相机由于原理限制, 无法在室外场景中很好地工作，环境中可能发生 物体和手的遮挡问题，这些都是值得探讨的问题.

对于虚拟/增强现实场景下的手部追踪问题， 一个独特的问题在于手大多数情况可能只有一部 分在视野中，甚至会丢失在视野中，而如何从这些 不完整的数据中恢复手部姿态也值得研究.

此外，在数据集的制作方面，用手动标记的方 法制作大样本数量的数据集成本过高，而合成的 数据集则丢失了深度图像的特征. 如何低成本地 获得高质量的、有标记的训练数据, 仍然没有很好 的解决方法.

本文认为, 未来手部姿态估计的研究在追求 准确性和鲁棒性的同时, 还需要考虑算法的泛化 能力和适用性等其他方面的因素，并考虑特定的 场景下可能带来的特殊问题.

\section{参考文献(References):}

[1] Li Jian, Tian Kun. Household of human-computer interaction in natural hand gestures recognition simulation accurately[J]. Computer Simulation, 2016, 33(11): 220-223(in Chinese) (李建, 田永. 家居中人机交互的自然手势准确识别仿真 $[\mathrm{J}]$. 计算机仿真, 2016, 33(11): 220-223)
[2] Wang R, Paris S, Popović J. 6D hands: markerless hand-tracking for computer aided design[C] //Proceedings of the 24th Annual ACM Symposium on User Interface Software and Technology. New York: ACM Press, 2011: 549-558

[3] Feng Zhiquan, Yang Bo, $\mathrm{Xu}$ Tao, et al. 3D direct human-computer interface paradigm based on free hand tracking[J]. Chinese Journal of Computers, 2014, 37(6): 1309-1323(in Chinese)

(冯志全, 杨波, 徐涛, 等. 基于自然手势跟踪的直接操作型 三维人机交互范式[J]. 计算机学报, 2014, 37(6): 1309-1323)

[4] Wu H Y, Wang J M. A visual attention-based method to address the midas touch problem existing in gesture-based interaction[J]. The Visual Computer, 2016, 32(1): 123-136

[5] Liang H, Wang J, Sun Q, et al. Barehanded music: real-time hand interaction for virtual piano[C] //Proceedings of the 20th ACM Symposium on Interactive 3D Graphics and Games. New York: ACM Press, 2016: 87-94

[6] Masakazu H, Takashi K. Robust finger tracking for gesture control of mobile devices using contour and interior information of a finger[J]. ITE Transactions on Media Technology and Applications, 2013, 1(3): 226-236

[7] Zhang Y, Meruvia-Pastor O. Operating virtual panels with hand gestures in immersive VR games[C] //Proceedings of International Conference on Augmented Reality, Virtual Re-ality and Computer Graphics. Heidelberg: Springer, 2017: 299-308

[8] Bilal S, Akmeliawati R, Salami M J E, et al. Vision-based hand posture detection and recognition for sign language - a study[C] //Proceedings of the 4th International Conference on Mechatronics. Los Alamitos: IEEE Computer Society Press, 2011: 1-6

[9] Kirac F, Kara Y E, Akarun L. Hierarchically constrained 3D hand pose estimation using regression forests from single frame depth data[J]. Pattern Recognition Letters, 2014, 50: 91-100

[10] Keskin C, Furkan K, Kara Y E, et al. Hand pose estimation and hand shape classification using multi-layered randomized decision forests[C] //Proceedings of European Conference on Computer Vision. Heidelberg: Springer, 2012: 852-863

[11] Verschaar M, Lobo D, Otaduy M A. Soft hand simulation for smooth and robust natural interaction[C] //Proceedings of the IEEE Conference on Virtual Reality and 3D User Interfaces. Los Alamitos: IEEE Computer Society Press, 2018: 183-190

[12] Holl M, Oberweger M, Arth C, et al. Efficient physics-based implementation for realistic hand-object interaction in virtual reality[C] //Proceedings of the IEEE Conference on Virtual Reality and 3D User Interfaces. Los Alamitos: IEEE Computer Society Press, 2018: 175-182

[13] Tian H, Wang C B, Manocha D, et al. Realtime hand-object interaction using learned grasp space for virtual environ-ments[J]. IEEE Transactions on Visualization and Computer Graphics, 2019, 25(8): 2623-2635

[14] Xiong Wei, Wang Qinghui, Li Jingrong. A hierarchical method of interaction hand gesture for virtual assembly[J]. Journal of South China University of Technology: Natural Science Edition, 2016, 44(1): 78-84(in Chinese)

(熊巍, 王清辉, 李静蓉. 面向虚拟装配的层次化交互手势 技术 $[J]$. 华南理工大学学报：自然科学版，2016，44(1): 78-84)

[15] Cai M, Kitani K M, Sato Y. An ego-vision system for hand grasp analysis[J]. IEEE Transactions on Human Machine Systems, 2017, 47(4): 524-535

[16] Faria D R, Trindade P, Lobo J, et al. Knowledge-based 
rea-soning from human grasp demonstrations for robot grasp synthesis[J]. Robotics and Autonomous Systems, 2014, 62(6): 794-817

[17] Feix T, Bullock I M, Dollar A M. Analysis of human grasping behavior: correlating tasks, objects and grasps[J]. IEEE Transactions on Haptics, 2014, 7(4): 430-441

[18] Bassily D, Georgoulas C, Guettler J, et al. Intuitive and adaptive robotic arm manipulation using the leap motion controller[C] //Proceedings of the 41st International Symposium on Robotics and German Conference on Robotics. Munich: VDE Press, 2014: 78-84

[19] Wang Xiaoyuan, Tian Hao, Wang Changbo. Research on natural grasp generation of the virtual hand[J]. Journal of Computer-Aided Design \& Computer Graphics, 2020, 32(9): 1502-1508(in Chinese)

(王晓媛, 田浩, 王长波. 虚拟手自然抓取动作生成研究 $[\mathrm{J}]$. 计算机辅助设计与图形学学报, 2020, 32(9): 1502-1508)

[20] Kim J H, Thang N D, Kim T S. 3-D hand motion tracking and gesture recognition using a data glove[C] //Proceedings of the IEEE International Symposium on Industrial Electronics. Los Alamitos: IEEE Computer Society Press, 2009: 1013-1018

[21] Kumar P, Rautaray S S, Agrawal A. Hand data glove: a new generation real-time mouse for human-computer interaction[C] //Proceedings of the 1st International Conference on Recent Advances in Information Technology. Los Alamitos: IEEE Computer Society Press, 2012: 750-755

[22] de Villiers H A C, van Zijl L, Niesler T R. Vision-based hand pose estimation through similarity search using the earth mover's distance[J]. IET Computer Vision, 2012, 6(4): 285-295

[23] Wang R Y, Popović J. Real-time hand-tracking with a color glove[J]. ACM Transactions on Graphics, 2009, 28(3): Article No.63

[24] Aristidou A. Hand tracking with physiological constraints[J]. The Visual Computer, 2018, 34(2): 213-228

[25] Erol A, Bebis G, Nicolescu M, et al. Vision-based hand pose estimation: a review $[\mathrm{J}]$. Computer Vision and Image Understanding, 2007, 108(1/2): 52-73

[26] Zhu Jiyu, Wang Xiying, Wang Weixin, et al. Hand gesture recognition based on structure analysis[J]. Chinese Journal of Computers, 2006, 29(12): 2130-2137(in Chinese)

(朱继玉, 王西颖，王威信，等. 基于结构分析的手势识别 [J]. 计算机学报, 2006, 29(12): 2130-2137)

[27] Piumsomboon T, Clark A, Billinghurst M, et al. User-defined gestures for augmented reality[C] //Proceedings of the $\mathrm{CHI}$ Conference on Human Factors in Computing Systems. New York: ACM Press, 2013: 955-960

[28] Krupka E, Vinnikov A, Bar-Hillel A, et al. Toward realistic hands gesture interface: keeping it simple for developers and machines[C] //Proceedings of the CHI Conference on Human Factors in Computing Systems. New York: ACM Press, 2017: 1887-1898

[29] Madadi M, Escalera S, Carruesco A, et al. Top-down model fitting for hand pose recovery in sequences of depth images[J]. Image and Vision Computing, 2018, 79: 63-75

[30] Glauser O, Wu S H, Panozzo D, et al. Interactive hand pose estimation using a stretch-sensing soft glove[J]. ACM Transactions on Graphics, 2019, 38(4): Article No.41

[31] Zhang H, Bo Z H, Yong J H, et al. Interaction Fusion: real-time reconstruction of hand poses and deformable objects in hand-object interactions[J]. ACM Transactions on Graphics,
2019, 38(4): Article No.48

[32] Romero J, Tzionas D, Black M J. Embodied hands: modeling and capturing hands and bodies together $[\mathrm{J}]$. ACM Transactions on Graphics, 2017, 36(6): Article No.245

[33] Roditakis K, Makris A, Argyros A A. Generative 3D hand tracking with spatially constrained pose sampling[C] //Proceedings of British Machine Vision Conference. Durham: BMVA Press, 2017: Article No.157

[34] Tan D J, Cashman T, Taylor J, et al. Fits like a glove: rapid and reliable hand shape personalization[C] //Proceedings of the IEEE Conference on Computer Vision and Pattern Recognition. Los Alamitos: IEEE Computer Society Press, 2016: 5610-5619

[35] Aristidou A, Lasenby J. Motion capture with constrained inverse kinematics for real-time hand tracking[C] //Proceedings of the 4th IEEE International Symposium on Communications, Control and Signal Processing. Los Alamitos: IEEE Computer Society Press, 2010: 1-5

[36] Chen X H, Wang G J, Zhang C R, et al. SHPR-Net: deep semantic hand pose regression from point clouds[J]. IEEE Access, 2018, 6: 43425-43439

[37] Wu Y M, Ji W, Li X, et al. Context-aware deep spatiotemporal network for hand pose estimation from depth images[J]. IEEE Transactions on Cybernetics, 2018, 50(2): 787-797

[38] Malik J, Abdelaziz I, Elhayek A, et al. HandVoxNet: deep voxel-based network for $3 \mathrm{D}$ hand shape and pose estimation from a single depth map[C] //Proceedings of the IEEE Computer Vision and Pattern Recognition. Los Alamitos: IEEE Computer Society Press, 2020: 7111-7120

[39] Li J, Wang Z F. Local regression based hourglass network for hand pose estimation from a single depth image[C] //Proceedings of the 24th IEEE International Conference on Pattern Recognition. Los Alamitos: IEEE Computer Society Press, 2018: 1767-1772

[40] Wan C D, Probst T, van Gool L, et al. Dense 3D regression for hand pose estimation[C] //Proceedings of the IEEE/CVF Conference on Computer Vision and Pattern Recognition. Los Alamitos: IEEE Computer Society Press, 2018: 5147-5156

[41] Che Y L, Song Y X, Qi Y. A novel framework of hand localization and hand pose estimation[C] //Proceedings of the IEEE International Conference on Acoustics, Speech and Signal Processing. Los Alamitos: IEEE Computer Society Press, 2019: 2222-2226

[42] Ting P W, Chou E T, Tang Y H, et al. Hand pose estimation based on 3D residual network with data padding and skeleton steadying[C] //Proceedings of Asian Conference on Computer Vision. Heidelberg: Springer, 2018: 293-307

[43] Krejov P, Bowden R. Multi-touchless: real-time fingertip detection and tracking using geodesic maxima[C] //Proceedings of the 10th IEEE International Conference and Workshops on Automatic Face and Gesture Recognition. Los Alamitos: IEEE Computer Society Press, 2013: 1-7

[44] Mueller F, Davis M, Bernard F, et al. Real-time pose and shape reconstruction of two interacting hands with a single depth camera[J]. ACM Transactions on Graphics, 2019, 38(4): Article No.49

[45] Krejov P, Gilbert A, Bowden R. Combining discriminative and model based approaches for hand pose estimation[C] //Proceedings of the 11th IEEE International Conference and Workshops on Automatic Face and Gesture Recognition. Los Alamitos: IEEE Computer Society Press, 2015: 1-7 
[46] Che Y L, Qi Y. Embedding gesture prior to joint shape optimization based real-time 3D hand tracking[J]. IEEE Access, 2020, 8: 34204-34214

[47] Tkach A, Tagliasacchi A, Remelli E, et al. Online generative model personalization for hand tracking $[\mathrm{J}]$. ACM Transactions on Graphics, 2017, 36(6): Article No.243

[48] Remelli E, Tkach A, Tagliasacchi A, et al. Low-dimensionality calibration through local anisotropic scaling for robust hand model personalization[C] //Proceedings of the IEEE International Conference on Computer Vision. Los Alamitos: IEEE Computer Society Press, 2017: 2554-2562

[49] Oberweger M, Wohlhart P, Lepetit V. Hands deep in deep learning for hand pose estimation[OL]. [2020-11-18]. https://arxiv.org/abs/1502.06807

[50] Zhou X Y, Wan Q F, Zhang W, et al. Model-based deep hand pose estimation[C] //Proceedings of the 25th International Joint Conference on Artificial Intelligence. New York: AAAI Press, 2016: 2421-2427

[51] Foix S, Alenya G, Torras C. Lock-in time-of-flight (ToF) cameras: a survey[J]. IEEE Sensors, 2011, 11(9): 1917-1926

[52] Zanuttigh P, Marin G, Mutto C D, et al. Operating principles of structured light depth cameras[M] //Time-of-Flight and Structured Light Depth Cameras. Heidelberg: Springer, 2016: 43-79

[53] Xu C, Cheng L. Efficient hand pose estimation from a single depth image[C] //Proceedings of the IEEE International Conference on Computer Vision. Los Alamitos: IEEE Computer Society Press, 2013: 3456-3462

[54] Liang H, Yuan J S, Thalmann D. Resolving ambiguous hand pose predictions by exploiting part correlations[J]. IEEE Transactions on Circuits and Systems for Video Technology, 2015, 25(7): 1125-1139

[55] Tompson J, Stein M, Lecun Y, et al. Real-time continuous pose recovery of human hands using convolutional networks[J]. ACM Transactions on Graphics, 2014, 33(5): Article No.169

[56] Oberweger M, Riegler G, Wohlhart P, et al. Efficiently creating $3 \mathrm{D}$ training data for fine hand pose estimation[C]//Proceedings of the IEEE Conference on Computer Vision and Pattern Recognition. Los Alamitos: IEEE Computer Society Press, 2016: 4957-4965

[57] Sridhar S, Oulasvirta A, Theobalt C. Interactive markerless articulated hand motion tracking using RGB and depth data[C] //Proceedings of the IEEE International Conference on Computer Vision. Los Alamitos: IEEE Computer Society Press, 2013: 2456-2463

[58] Rogez G, Supancic J S, Khademi M, et al. 3D hand pose detection in egocentric RGB-D images[C] //Proceedings of European Conference on Computer Vision Workshop. Heidelberg: Springer, 2014: 356-371

[59] Qian C, Sun X, Wei Y C, et al. Realtime and robust hand tracking from depth[C]//Proceedings of the IEEE Conference on Computer Vision and Pattern Recognition. Los Alamitos: IEEE Computer Society Press, 2014: 1106-1113

[60] Tang D, Yu T H, Kim T K. Real-time articulated hand pose estimation using semi-supervised transductive regression forests[C] //Proceedings of the IEEE International Conference on Computer Vision. Los Alamitos: IEEE Computer Society Press, 2013: 3224-3231

[61] Sun X, Wei Y C, Liang S, et al. Cascaded hand pose regression[C] //Proceedings of the IEEE Conference on Computer Vision and Pattern Recognition. Los Alamitos: IEEE Computer
Society Press, 2015: 824-832

[62] Sharp T, Keskin C, Robertson D, et al. Accurate, robust, and flexible real-time hand tracking[C] //Proceedings of the ACM Conference on Human Factors in Computing Systems. New York: ACM Press, 2015: 3633-3642

[63] Wetzler A, Slossberg R, Kimmel R. Rule of Thumb: deep derotation for improved fingertip detection[C] //Proceedings of the British Machine Vision Conference. Durham: BMVA Press, 2015: Article No.33

[64] Sridhar S, Mueller F, Zollhöfer M, et al. Real-time joint tracking of a hand manipulating an object from RGB-D input[C] //Proceedings of European Conference on Computer Vision. Heidelberg: Springer, 2016: 294-310

[65] Zhang J W, Jiao J B, Chen M L, et al. A hand pose tracking benchmark from stereo matching[C]//Proceedings of the IEEE International Conference on Image Processing. Los Alamitos: IEEE Computer Society Press, 2017: 982-986

[66] Zimmermann C, Brox T. Learning to estimate 3D hand pose from single RGB images[C] //Proceedings of the IEEE International Conference on Computer Vision. Los Alamitos: IEEE Computer Society Press, 2017: 4913-4921

[67] Mueller F, Mehta D, Sotnychenko O, et al. Real-time hand tracking under occlusion from an egocentric RGB-D sensor[C] //Proceedings of the IEEE International Conference on Computer Vision Workshops. Los Alamitos: IEEE Computer Society Press, 2018: 1284-1293

[68] Yuan S X, Ye Q, Stenger B, et al. BigHand2.2M benchmark: hand pose dataset and state of the art analysis[C] //Proceedings of the IEEE Conference on Computer Vision and Pattern Recognition. Los Alamitos: IEEE Computer Society Press, 2017: 2605-2613

[69] Garcia-Hernando G, Yuan S X, Baek S, et al. First-person hand action benchmark with RGB-D videos and 3D hand pose annotations[C] //Proceedings of the IEEE Conference on Computer Vision and Pattern Recognition. Los Alamitos: IEEE Computer Society Press, 2018: 409-419

[70] Tkach A, Pauly M, Tagliasacchi A. Sphere-meshes for real-time hand modeling and tracking[J]. ACM Transactions on Graphics, 2016, 35(6): Article No.222

[71] Tagliasacchi A, Schröder M, Tkach A, et al. Robust articulated-ICP for real-time hand tracking[J]. Computer Graphics Forum, 2015, 34(5): 101-114

[72] Choi C, Kim S, Ramani K. Learning hand articulations by hallucinating heat distribution[C] //Proceedings of the IEEE International Conference on Computer Vision. Los Alamitos: IEEE Computer Society Press, 2017: 3123-3132

[73] Oikonomidis I, Kyriazis N, Argyros A. Efficient model-based $3 \mathrm{D}$ tracking of hand articulations using Kinect[C] //Proceedings of the British Machine Vision Conference. Durham: BMVA Press, 2011: 101

[74] Shotton J, Girshick R, Fitzgibbon A, et al. Efficient human pose estimation from single depth images[J]. IEEE Transactions on Software Engineering, 2013, 35(12): 2821-2840

[75] Shotton J, Fitzgibbon A, Cook M, et al. Real-time human pose recognition in parts from single depth images[C] //Proceedings of the IEEE Conference on Computer Vision and Pattern Recognition. Los Alamitos: IEEE Computer Society Press, 2011: 1297-1304

[76] Zhang Z Y. Microsoft Kinect sensor and its effect[J]. IEEE 
Multimedia, 2012, 19(2): 4-10

[77] Taylor J, Bordeaux L, Cashman T, et al. Efficient and precise interactive hand tracking through joint, continuous optimization of pose and correspondences[J]. ACM Transactions on Graphics, 2016, 35(4): Article No.143

[78] Ye Q, Yuan S X, Kim T K. Spatial attention deep net with partial PSO for hierarchical hybrid hand pose estimation[C] //Proceedings of European Conference on Computer Vision. Heidelberg: Springer, 2016: 346-361

[79] Fleishman S, Kliger M, Lerner A, et al. ICPIK: inverse kinematics based articulated-ICP[C] //Proceedings of the IEEE Conference on Computer Vision and Pattern Recognition Workshops. Los Alamitos: IEEE Computer Society Press, 2015: 28-35

[80] Melax S, Keselman L, Orsten S. Dynamics based 3D skeletal hand tracking[C] //Proceedings of the ACM SIGGRAPH Symposium on Interactive 3D Graphics and Games. New York: ACM Press, 2013: 63-70

[81] Che Y L, Qi Y. Dynamic projected segmentation networks for hand pose estimation[C]//Proceedings of the 24th International Conference on Pattern Recognition. Los Alamitos: IEEE Computer Society Press, 2018: 477-482

[82] Sridhar S, Mueller F, Oulasvirta A, et al. Fast and robust hand tracking using detection-guided optimization[C] //Proceedings of the IEEE Conference on Computer Vision and Pattern Recognition. Los Alamitos: IEEE Computer Society Press, 2015: 3213-3221

[83] Sridhar S, Rhodin H, Seidel H, et al. Real-time hand tracking using a sum of anisotropic Gaussians model[C] //Proceedings of the 2nd International Conference on 3D Vision. Los Alamitos: IEEE Computer Society Press, 2014: 319-326

[84] Khamis S, Taylor J, Shotton J, et al. Learning an efficient model of hand shape variation from depth images[C] //Proceedings of the IEEE Conference on Computer Vision and Pattern Recognition. Los Alamitos: IEEE Computer Society Press, 2015: 2540-2548

[85] Sinha A, Choi C, Ramini K. DeepHand: robust hand pose estimation by completing a matrix imputed with deep features[C] //Proceedings of the IEEE Conference on Computer Vision and Pattern Recognition. Los Alamitos: IEEE Computer Society Press, 2016: 4150-4158

[86] Yang H W, Zhang J Y. Hand pose regression via a classification-guided approach[C] //Proceedings of Asian Conference on Computer Vision. Heidelberg: Springer, 2016: 452-466

[87] Tang D, Chang H J, Tejani A, et al. Latent regression forest: structured estimation of 3D hand poses[J]. IEEE Transactions on Pattern Analysis and Machine Intelligence, 2017, 39(7): 1374-1387

[88] Oberweger M, Wohlhart P, Lepetit V. Training a feedback loop for hand pose estimation[C] //Proceedings of the IEEE International Conference on Computer Vision. Los Alamitos: IEEE Computer Society Press, 2015: 3316-3324

[89] Ge L H, Liang H, Yuan J S, et al. Robust 3D hand pose estimation in single depth images: from single-view CNN to multi-view CNNs[C] //Proceedings of the IEEE Conference on Computer Vision and Pattern Recognition. Los Alamitos: IEEE Computer Society Press, 2016: 3593-3601
[90] Ge L H, Cai Y J, Weng J W, et al. Hand PointNet: 3D hand pose estimation using point sets[C] //Proceedings of the IEEE/CVF Conference on Computer Vision and Pattern Recognition. Los Alamitos: IEEE Computer Society Press, 2018: 8417-8426

[91] Ge L H, Liang H, Yuan J S, et al. 3D convolutional neural networks for efficient and robust hand pose estimation from single depth images[C] //Proceedings of the IEEE Conference on Computer Vision and Pattern Recognition. Los Alamitos: IEEE Computer Society Press, 2017: 5679-5688

[92] Chang J Y, Moon G, Lee K M. V2V-PoseNet: voxel-to-voxel prediction network for accurate $3 \mathrm{D}$ hand and human pose estimation from a single depth map[C] //Proceedings of the IEEE/CVF Conference on Computer Vision and Pattern Recognition. Los Alamitos: IEEE Computer Society Press, 2018: 5079-5088

[93] Chen Y J, Tu Z G, Ge L H, et al. SO-HandNet: self-organizing network for $3 \mathrm{D}$ hand pose estimation with semi-supervised learning[C] //Proceedings of the IEEE International Conference on Computer Vision. Los Alamitos: IEEE Computer Society Press, 2019: 6960-6969

[94] Baek S, Kim K I, KimT K. Weakly-supervised domain adaptation via GAN and mesh model for estimating 3D hand poses interacting objects[C] //Proceedings of the IEEE/CVF Conference on Computer Vision and Pattern Recognition. Los Alamitos: IEEE Computer Society Press, 2020: 6120-6130

[95] Neverova N, Wolf C, Nebout F, et al. Hand pose estimation through semi-supervised and weakly-supervised learning[J]. Computer Vision and Image Understanding, 2017, 164: 56-67

[96] Wan C D, Probst T, van Gool L, et al. Self-supervised 3D hand pose estimation through training by fitting[C] //Proceedings of the IEEE/CVF Conference on Computer Vision and Pattern Recognition. Los Alamitos: IEEE Computer Society Press, 2019: 10845-10854

[97] Doosti B, Naha S, Mirbagheri M, et al. HOPE-Net: a graph-based model for hand-object pose estimation[C] //Proceedings of the IEEE Conference on Computer Vision and Pattern Recognition. Los Alamitos: IEEE Computer Society Press, 2020: 6607-6616

[98] Krejov P, Gilbert A, Bowden R. Guided optimisation through classification and regression for hand pose estimation[J]. Computer Vision and Image Understanding, 2017, 155: 124-138

[99] Taylor J, Tankovich V, Tang D H, et al. Articulated distance fields for ultra-fast tracking of hands interacting[J]. ACM Transactions on Graphics, 2017, 36(6): Article No.244

[100] Shelhamer E, Long J, Darrell T. Fully convolutional networks for semantic segmentation[J]. IEEE Transactions on Pattern Analysis and Machine Intelligence, 2017, 39(4): 640-651

[101] Davison A J, Reid I D, Molton N D, et al. MonoSLAM: real-time single camera SLAM[J]. IEEE Transactions on Pattern Analysis and Machine Intelligence, 2007, 29(6): 1052-1067

[102] Wang G J, Chen X H, Guo H K, et al. Region ensemble network: towards good practices for deep 3D hand pose estimation[J]. Journal of Visual Communication and Image Representation, 2018, 55: 404-414 